\title{
Yoga - Meditation and Positive Psychology
}

Jyotsna Agrawal, Associate Professor, Department of Clinical Psychology

Faculty in-Charge, VIPRA (Vedic Indian Psychology Research \& Application)

NIMHANS, Bangalore jyotsna.agl@gmail.com

\subsection{Objectives:}

1.1. To recognize the role yoga-meditation plays in positive mental health

1.2. To understand the various meanings of yoga

1.3. To learn about Patanjali's yoga

1.4. To study about meditations, its types and its relationship with yoga

\subsection{Introduction}

The Positive Psychology movement has been a recent development in the short history of psychology, a journey of fewer than 200 years. Its scope is present in the context of the global burden of mental health disorders. Studies indicate that mental disorders are one of the primary causes of years lost due to disability (YLD) or suicide (Reddy, 2010). Currently, more than 350 million people worldwide suffer from depression and as per WHO, by 2020 depression will be the second leading cause of disease (WHO, Global Health Estimates: Life expectancy and leading causes of death and disability, 2010). Even in India, Depressive disorders have a prevalence rate of 5.1\% (SURVEY, 2015-16).

Nevertheless, routine mental health services are limited, and people continue to suffer even when various empirically validated therapeutic interventions are present. Many such people do not seek treatment (due to stigma, lack of finances, etc.) For others, the conventional treatment is not optimal due to mismatches related to personality, goals, values, resources and lifestyles. Further, approaches for the acute phase treatment may not work to prevent relapse, and there 
is a need to limit or remove the residual symptoms. Additionally, beyond symptom recovery, psychological recovery needs to be given attention (Bockting, 2015; Hofmann, 2010; Huhn, 2014; Layous, 2011).

Although the definition of mental health includes positive mental health and well-being (WHO, 2001), its promotion requires a different approach. Here the role of positive psychology is significant, and it can provide various insights for positive mental health interventions. Various studies have shown that positive psychology based interventions buffer against stress, improve health and productivity, and enhance social connectedness (Vázquez, 2009).

However, there has been some criticism of positive psychology leading to second wave positive psychology (Lomas T. \&., 2016). For example, it may seem too optimistic and wishful; or be equated with only happiness and seem to ignore the value of some negative emotions. The one

criticism which seems severe and worth delving into deeper is that positive psychology ignores a multi-cultural perspective. Studies have shown that positive psychology interventions work better in individualistic cultures, where the cultural norms support the pursuit of individual happiness (Suh, 2008; Sin, 2009).

\subsection{Need to study yoga and meditation traditions for positive mental health}

The values and assumptions based on which people find answers to 'why' and 'how' questions, i.e., meaning in life and the right way to live, is influenced by culture. These ideas are deeprooted and are not choices but work below the surface of awareness. Since positive psychology has developed in western culture, it has an underlying lens of individualism (Christopher, 2008). The third wave of positive psychology has emerged in response to this criticism, which emphasises cultural components (Lomas, et. al, 2020). Indian culture has a rich philosophicalpsychological heritage and a different perspective on human life and its ultimate goal. 
Therefore, it would be apt to explore indigenous ideas of positive mental health, which seems to be closely related to yoga and meditation. A stack of studies has already been accumulated, indicating various benefits of yoga and meditation for both mind and body. Hence it is relevant to understand yoga and meditation in their totality, which will be attempted in this chapter.

\section{Self-Assessment Questions}

Q1. As per WHO, ......... Will be the second leading cause of disease by 2020.

Q2. The prevalence of Depressive disorders in India is less than 5\%. - True/ False

Q3. Positive psychology has an underlying individualism - True/ False

Q4. The ... wave of positive psychology emphasises culture.

\subsection{What is Yoga}

Living with a deep and permanent sense of well-being, along with finding freedom from suffering, can seem like an unachievable goal. However, this is the promise of yoga if we are willing to sincerely walk on the path of yoga in its complete form. In recent times yoga is often perceived as synonymous with a combination of asanas (bodily postures) and pranayama (breathing exercises), along with the inclusion of meditation (Goyal, et al., 2014). Nevertheless, that has not been the meaning (Layous, 2011) of yoga as explained over the centuries in the Indian civilisation, where it is a set of practices to access higher Consciousness and states of being. Yoga literally means to yoke, from the Sanskrit root yuj, which means to join and refers to experiencing oneness with the Supreme Consciousness. The Encyclopedia of Indian Philosophies Volume XII, Yoga: India's Philosophy of Meditation (Larson and Bhattacharya, 2016), defines yoga in two ways. First, yoga means all those techniques people practice to find release from their existential suffering and achieve unity 
consciousness. Second, yoga refers to a specific school of Indian philosophy given by Patanjali in his text Yogasutra.

Thus, to fully appreciate its role in positive mental health, we need to explore yoga deeply, its aims and related assumptions. One of the core assumptions is related to how the world is made of not just matter but has underlying Consciousness. Based on this idea, yogic practices aim towards experiencing pure Consciousness or some similar higher state considered as liberation in a specific yogic system. A variety of terms have been used for such liberated states, such as Kaivalya, Mukti etc. This liberation is not a transitory state experienced only during deep meditation but a complete transformation of one's ordinary living, working and relating to others (Agrawal \& Cornelissen, 2021).

\subsection{The three major Yogic paths}

While exploring, you may come across a variety of yogic systems. The trimarga, or the three main yogic pathways of bhakti, jnana, and karma yoga, is a standard method to categorise these multiple styles and systems. These three pathways are discussed next (Agrawal \& Cornelissen, 2021).

3.1.1. Bhakti yoga: the yogic path of bhakti focuses on the cultivation of emotions around the Divine and supposedly results in bliss when one achieves oneness with one's preferred form of God. In the process, one lets go of ego and desire-based lifestyle. This path may seem to be familiar and probably most accessible. Thus, one may note a large number of bhakti saints revered in different parts of the country.

3.1.2. Jnana yoga: the Jnanayoga path is that of cognitive realisation of the oneness of consciousness, which may later be experienced first-hand and experientially. Here again, one 
may drop one's biases and note the reality as it is, thus removing many sources of pain and suffering.

3.1.3. Karma yoga: this path, most famously described in Bhagavad Gita, is associated with the proper use of one's volition or willpower. This path's core is working without desires (nishkama karma) and not being attached to specific outcomes, thereby finding inner freedom. One does work with one's utmost capacities, skilfully, and with an attitude of fulfilling one's duties for the larger good of the world. Even when one engages in one's regular affairs, this path too can lead to ultimate freedom, as discussed in other yogic paths.

It is relevant to note that most people do not practice these yogic paths in an isolated manner. Instead, they may mix components, with some or other aspect being dominant, indicating their own personal synthesis. Even yogis and mystics combine these paths in their discourses. Yogiphilosopher Sri Aurobindo has given Integral Yoga, with a synthesis of these paths not just in application but also in their philosophy in his book Synthesis of Yoga.

\section{Self-Assessment Questions}

Q5. Yoga means techniques to find ....

Q6. The trimarga yoga are, ... yoga .... yoga and .... yoga.

Q7. Cultivation of emotions around the Divine is known as Karma yoga - True/ False

Q8. The cognitive realisation of the oneness of consciousness is Jnana yoga - True/ False Q9. Karma yoga has been extensively described in .... text.

\subsection{Patanjali's Yoga}

As mentioned, one way to understand yoga is to follow Patanjali's Yogasutra. The text Yogasutra as codified by Patanjali is a millennia old text from India, and evidence from archaeology, various other texts, and oral tradition indicate that this yogic system is probably 
more than 5000 years old. It describes yoga philosophy, steps and outcomes, and has many ancient and modern commentaries written on it. There are 195 Sanskrit verses (known as Sutras) in Patanjali Yogasutra, categorised in 4 chapters (Pada) to explain profound ideas in the philosophy of mind and its applications. While yoga means union, sutra denotes thread and formula both; thus, Yogasutra may mean a thread of formulas codifying methods to achieve union with the highest Consciousness. These Sutras are short and crisp, meant to be learnt byheart, chanted and contemplated upon easily, since these were written when printing was either unavailable or expensive and time-consuming. This system is also termed Raja-yoga, roughly translated as the king of yoga (Taimini, 1961).

\subsubsection{The four chapters of Patanjali Yogasutra}

While first (Samadhi Pada) and last (Kaivalya Pada) chapters are focused upon advanced states of meditation (Samadhi) and ultimate liberation (Kaivalya). The second (Sadhana Pada) and third (Vibhuti Pada) chapters provide insights into the process of yoga practice. The chapters are elaborated on next.

3.2.1.1. Samadhi Pada (Chapter 1, 51 sutras): This chapter discusses the definition of yoga as stilling of the five types of activities of mind and identifying with deeper awareness. This process occurs through a disciplined effort and detachment. It further discusses various types and levels of samadhi, which is a deep absorptive state. Some essential aids on this path are faith, courage and memory (of past states). The intensity of motivation also counts, and for an intensely focused student, the realisation happens quickly. While Iswara/ Divine is consciousness without any afflictions, others have five types of afflictions (klesha) which lead to suffering. Chanting of OM can connect us to the Supreme Consciousness (Iswara).

Additionally, nine obstacles on this spiritual journey and four signs of the same are also mentioned. Various techniques one can use to overcome these obstacles and thereby 
silence the mind has also been suggested. The chapter describes a few higher types of samadhi and how the stilling of the mind will reflect true knowledge in pure awareness. 3.2.1.2. Sadhana Pada (Chapter 2, 55 sutras): This chapter starts with discussing Kriya yoga, a combination of self-study (svadhyaya), disciplined endurance (tapa) and surrender to Supreme Consciousness (Ishvara-pranidhana). This process of kriya yoga is a means to remove five afflictions (klesha) and gradually get freedom from laws of action-reaction (Karma). The Ashtanga yoga, an eight-limbed path of yoga, is subsequently introduced with limbs of Yama, Niyama, Asana, Pranayama, Pratyahara, Dharana, Dhyana and Samadhi. These limbs are further categorised as external and internal. Together they are meant to overcome present misery and avoid future suffering.

3.2.1.3. Vibhuti Pada (Chapter 3, 56 sutras): This chapter discusses the last three steps of Ashtanga yoga, i.e., Dharana, dhyana and samadhi. When these are practised together, it is called Samyama. As per Patanjali, extraordinary knowledge and powers may be gained through samyama. The usefulness of vairagya and the need for purification of the mind is also emphasised in this chapter.

3.2.1.4. Kaivalya Pada (Chapter 4,34 sutras): This is the final chapter of the Patanjali yoga sutra, where the state of liberation (kaivalya) is discussed in detail, along with various extraordinary capacities. Additionally, the law of karma, rebirth, gunas, ordinary perception versus higher discrimination is discussed. The chapter ends with an introduction of the highest samadhi state known as Dharma-megha samadhi.

\section{Self-Assessment Questions}

Q10. Patanjali's Yogasutra, has 195 sutras divided in .... number of chapters.

Q11. Chanting of OM as a technique is given in Samadhi pada. - True/ False 
Q12. Kriya yoga has been discussed in Kaivalya pada. - True/ False

Q13. Dharmamegha samadhi, the highest kind of meditative state has been described in Kaivalya Pada. - True/ False

Q14. Kriya yoga has three components of and Ishvara-pranidhana.

Q15. The eight limbs of Ashtanga yoga are divided into two parts, bahiranga and ....

\subsubsection{Important ideas in Patanjali Yogasutra}

3.2.2.1. Definition of yoga: Patanjali Yogasutra begins by providing a definition of yoga as the process of silencing the mind, by stopping the waves of mental activities known as Chittavrittis. The five kinds of mental activities / chitta-vrittis which needs silencing are: (a) Realitybased thinking, (b) Misconceptions, (c) Imaginations of future, (d) Memory of past, and (e) Sleepy or dull states.

3.2.2.2. Process of yoga: The mind of a yogi, which is a calm and silent mind may seem unrealistic, but it can be achieved through: (a) Constant practice (Abhyasa), (b) Detachment (Vairagya). Certain other helpful psychological aids are also mentioned: ((i) Faith (Shraddha), (ii) Courage and strength (Virya), (iii) Memory and learning (Smriti), and (iv) Wisdom associated with higher states (Samadhi Prajna).

The role of motivation and effort has further been emphasised. It interacts with different types of seekers (dull, average or competent), resulting in varying speeds of accomplishments.

3.2.2.3. Barriers in the yoga practice: On our yogic journey to liberation, nine hurdles and barriers have been enumerated by Patanjali. These are: (a) Illness, (b) Dullness, (c) Doubt, (d) Distraction, (e) Laziness, (f) Desire and craving, (g) Imagination and fantasy, (h) Lack of progress, (i) Lost accomplishments.

These nine barriers can overtly express themselves in terms of four signs: (i) Suffering, (ii) Despair, (iii) Bodily instability, and (iv) Irregular breathing. Under the unhealthy influence of 
greed, anger and/ or delusions, one may engage in negative behaviour, either directly by doing something negative or indirectly by making others do something negative or approve a negative behaviour.

3.2.2.4. Overcoming the barriers in the yoga practice: To purify and stabilise our mind and overcome negative tendencies which can act like obstacles against further progress on the yogic path, one can utilise various methods: (a) Develop single-minded practice, (b) Developing the qualities of unconditional friendliness and loving-kindness and (Maitri), compassion (Karuna), sympathetic and appreciative joy (Mudita), and non-reactivity and equanimity (Upeksha). (c) Practice focusing of attention (on breath/ on one's sensory experiences/ on internal light). (d) Cultivating detachment, (e) Learning from dream analysis, and (f) Meditations.

Apart from these, one can also practice the development of opposite thoughts and feelings to bring balance, known as Pratipaksha Bhavana. For example, when one has criticality towards another person, one can try to develop an appreciation for the same person.

3.2.2.5. Role of Ishwara / Divine in Yoga practice: The term Ishwara/ Divine, denotes 'That Special Soul' (Vishesha Purusha), which is beyond afflictions (Kleshas) and laws of actionreaction (Karma) and is the timeless source of wisdom which can guide us as our eternal teacher. Pranava (AUM or Om) is the sound of Supreme Consciousness (Iswara), where the sound of A represents creation, $\mathrm{U}$ denotes maintenance, and $\mathrm{M}$ represents dissolution aspects. It has healing properties, and its recitation (Japa) can also remove barriers from the yogic path. 3.2.2.6. Klesha model of suffering: One of the important models on psychological causes of suffering as presented by Patanjali is the following five core afflictions (Klesha):

a) Avidya - which is the primary ignorance of our true Self, and it is described to be an error where one believes the non-eternal to be everlasting, the impure as pure, misunderstands suffering as happiness, and non-self to be the Self. 
b) Asmita - it is developed next when one misunderstands the mind to be our true Self.

c) Raga - it is the attachment to pleasure.

d) Dwesha - it is the aversion to pain.

e) Abhinivesha - the desire for living and fear of death is the fifth affliction.

At any point in time, these five afflictions can be either dormant, attenuated, manifest or powerful, and when active, they can keep us in the cycle of action-reaction (Karma).

3.2.7. Overcoming Klesha: To overcome these afflictions and to avoid future suffering, one needs to realise the true Self as separate from the material world made up of Triguna, i.e., the principles of Sattva/ light, Rajas/ movement and Tamas/ inertia.

One can overcome afflictions by the practice of Kriyayoga, i.e., practicing self-study (svadhyaya), disciplined endurance (tapa) and surrender to Supreme Consciousness (Ishvarapranidhana). These steps are also part of Niyama, one of the eight limbs of Ashtanga yoga, which are described in next section.

\section{Self-Assessment Questions}

Q16. The five kinds of chitta-vrittis are: (a) Reality-based thinking, (b) Misconceptions, (c) Imaginations of future, (d) Memory of past, and (e) Super active states. - True/ False Q17. We need constant practice (abhyasa) and .... for yoga.

Q18. The nine barriers in yoga practice, express themselves as: suffering, ....., bodily instability, and

Q19. Development of opposite thoughts and feelings to bring mental balance is known as .... Q20. In OM sound, A represents ...., $\mathrm{U}$ denotes ...., and $\mathrm{M}$ represents .... Q21. The five Klesha are avidya, ....,....,.... and abhinivesha. Q22. Abhinivesha means the desire for living and fear of death - True/ False 
3.2.3. The Ashtanga (eight-limbed) yoga of Patanjali: The most famous contribution of Patanjali is his comprehensive system of Ashtanga yoga. It not only purifies the mind, but also helps in experiencing the state of deepest absorption or samadhi. These eight limbs are further categorised into external (Bahiranga yoga) and internal (Antaranga yoga), depending on the focus of these practices. (a) Bahiranga yoga: Yama, Niyama, Asana, Pranayama. The fifth limb Pratyahara although grouped with bahiranga, is a bridge with the antaranga components of ashtanga yoga. (b) Antaranga yoga: Dharana, Dhyana and Samadhi.

3.2.3.1. Yamas: The five yamas emphasise ethics in one's relations to the outer world, not only in actions but also in speech and thoughts. These yamas are:
a) Non-harming (Ahimsa)
b) Truthfulness (Satya)
c) Non-stealing (Asteya)
d) Following the highest truth (Brahmacharya), and
e) Non-possessiveness (Aparigraha)

3.2.3.2. Niyamas: The five Niyamas are focused on our relationship to ourselves. These are:
a) Purity of body and mind (Saucha)
b) Contentment (Santosha)
c) Highest discipline (Tapa)
d) Study and analysis of oneself (Svadhyaya), and
e) Surrender to the Divine (Ishwara Pranidhana)

The first two limbs of Yama-Niyama are the foundations of the practice, and when they are consistently practised beyond consideration for individual and contextual variations, such as that of place, time, context or family lineage etc., these can become the great vows (Mahavrata). 
Special powers and capacities may develop when any of these Yamas and Niyamas are established within a person as a great vow through unwavering practice. The last three Niyama of Tapa, Svadhyaya and Ishwara-pranidhana is also known as Kriya-yoga and can remove five afflictions. As per Patanjali, even merely the practice of last Niyama (Ishwara-pranidhana) can lead to the highest Samadhis.

3.2.3.3. Asana: Asana is described as that which leads to comfortable stability (sthira sukham asanam - PYS 2.46). When asana is practised meditatively without efforts, one can experience equanimity and non-duality.

3.2.3.4. Pranayama: Pranayama is described as the stopping of inhalation and exhalation, which can improve attention and prepare the mind for advanced yoga. A few types of Pranayama are discussed, accompanying with how the process can be influenced by factors like location, season etc. Although the components of Asana-Pranayama are most emphasised in the modern world, one may notice Patanjali has not emphasised these so much.

3.2.3.5. Pratyahara: Pratyahara is disengaging and withdrawing one's mind from the external, sensory objects of attention.

3.2.3.6. Dharana: Dharana is the act of holding attention to a single object or point.

3.2.3.7. Dhyana: When this one-pointed attention becomes steady and unbroken, it is called Dhyana.

3.2.3.8. samadhi: When the state of deep absorption is reached, where only the object of attention is left and not the mind, it is known as samadhi.

When the limbs of Dharana, Dhyana and Samadhi are practised together, it is known as Samyama. The practice of Samyama on various objects or ideas can bring in a variety of knowledge and powers, as described in the Yogasutras.

\section{Self-Assessment Questions}


Q24. Antaranga yoga are Dharana,....... and Samadhi.

Q25. The five yamas are: ahimsa, satya, ..., brahmacharya, and ....

Q26. The five niyamas are Saucha, ....,...., svadhyaya and Ishwara pranidhana.

Q27. Asana is an uncomfortable stable pose - True/ False

Q28. Pranayama is described as the ongoing of inhalation and exhalation - True/ False

Q29. Disengaging one's mind from the external, sensory objects of attention is ....

Q30. When dharana, dhyana and samadhi are practised together, it is known as ....

\subsection{What is Meditation}

Meditation is an English term with Latin roots (meditari; to think, reflect or contemplate) and indicate 'deep exploration of mind' (Goleman \& Davidson, 2017). It is considered a 'technique to control the mind' (Rao \& Paranjpe, 2016) by controlling our attention and awareness (Walsh \& Shapiro, 2006). The process of meditation goes from attending to an object (Dharana), sustaining this attention in an unbroken manner (Dhyana) to complete absorption into it (Samadhi). Another term Bhavana is also used in Patanjali Yogasutra for cultivating maitri, mudita, karuna and upeksha. Meditation aims towards a higher state of consciousness. As byproducts of this process, healing and developing special powers are noted. The various definitions of meditation may not fully capture its essence due to the limitations of language. The US Department of Health and Human Services reached a similar conclusion regarding challenges in developing a taxonomy of meditation. Using expert consensus, (Cardoso, deSouza, Camano, \& Leite, 2004) tried to capture the meaning of meditation through five core criteria based upon its process and outcomes, to overcome some of these limitations. These were - it is done by oneself, using a technique, by relaxing the body, and the analytic mind, while focusing on oneself. Other relevant aspects of meditation were found to be an spiritualphilosophical context, mental silence and possibility of an altered consciousness. 
Many of the currently popular meditation techniques originated in the Indian civilization thousands of years back. It was introduced to the West in 1920 when Indian monk Paramahansa Yogananda travelled to USA. Around the 1970s, meditation got scientific attention when another Indian monk from the Himalayan tradition Swami Rama demonstrated voluntary control over multiple bodily organs in USA. Within a lab setting, he changed brain waves, heart functioning and skin temperature, which were known to be entirely automatic processes. Subsequently, American Psychological Association (1977) issued a statement noting that "meditation may facilitate the psychotherapeutic process" and also encouraging further research "to evaluate its possible usefulness" (as cited by Kutz, Borysenko and Benson, 1985).

\subsection{Broad Categories of Meditation}

For ease of research and understanding, a broad categorization that is often used lately has been mindfulness and concentration meditation, based on the differences in the attention process (Thomas \& Cohen, 2014; Lippelt, Hommel, \& Colzato, 2014).

4.1.1. Focused Attention / Concentrative Meditation: In this category of meditation, we focus all attention on a single point / object / idea/ image / sound. This meditation process is described in Dharana-Dhyana stages of Patanjali's ashtanga yoga, where we develop an unbroken flow of attention towards a single point. The process also includes removing attention from other distractions, as done in pratyahara stage. It also includes noticing when one has got distracted internally, by one's own thoughts and bringing attention back to the focus of attention. This process of meditation is also known as Samatha meditation in Buddhism. This type of meditation further travelled in Asia and developed regional variations and names in interaction with local culture and spirituality.

4.1.2. Mindfulness / Open Awareness / Open Monitoring Meditation: This ancient meditation from India was rediscovered and popularised through his teachings by Buddha. It 
is called Vipashyana, a unique way of seeing, and gaining insight and Sakshi bhava, which means the cultivation of being a witness. In this category of meditation, we try to keep an open and curious attitude towards whatever is happening in the body or mind. We remain aware of our thoughts, feelings and sensations in the present moment. When we notice these without any judgment or need for control, we can then let them go. In recent times the most popular way of learning the traditional form of Vipashyana around the world is through the lineage of Shri S.N. Goenka. Apart from up to 11 hours of daily meditation these retreats have video lectures on Buddhist philosophy related to suffering and release from the same. The aim is to develop an experiential insight into the non-permanent nature of our self and identity.

4.1.3. Transcendental Meditation: It is sometimes considered the third category of meditation. Maharshi Mahesh Yogi is acknowledged to be the founder of this meditation technique, who developed it in 1958 . Here we may start with silently reciting a mantra, which is usually a short, monosyllabic sound. Often though not always, these sounds are sacred beejmantra. However, in the process of meditating, we are supposed to transcend it or go beyond it. Here the mantra recitation process is connected with the state of mind, with increase in mantra recitation, when we encounter more distracting thoughts.

4.1.4. Interpersonally Oriented Meditations: These were a recently added category, and find their roots in Maitri or Metta meditation which is present in multiple traditions from ancient India (Agrawal \& Sahota, 2021). Here we try to cultivate loving-kindness, friendliness, goodwill and compassion for others. These qualities are supposed to be cultivated in a limitless manner, without personal preferences or aversion and thus are called the four Immeasurable. The process includes focusing on an idea, and seems to be a combination of concentration and mindfulness meditation practices.

\subsection{Other forms of meditations}


4.2.1. Japa/ Mantra Meditation: here we repeat a sacred word or phrase (mantra), either silently or with sound, for a pre-decided number of rounds or for a specific duration of time. This has components of Dhyana or concentrative meditation. The focus of attention is on the meaning of the mantra and its sound, and ignoring both external and internal distractions. As mentioned earlier, Transcendental meditation also uses a mantra, but instead of focusing on the mantra itself, the aim is to transcend it (Lynch, et al., 2018).

4.2.2. Yoga Nidra, Shavasana: In these types of meditations, we sequentially relax our bodies. Additionally, there may be specific visualization included. In Yoga Nidra, one also attempts to develop a witnessing quality.

4.2.3. Other Common Meditation Practices: A few other commonly practiced meditations are related to body or breath, such as scanning the body for various sensations or focusing attention on one's breathing. Another type of meditation is related to Kundalini, where one tries to awaken and uplift the psycho-spiritual energy in one's body. Yogasana when done mindfully with rhythmic breaths is a type of movement meditation, and other such types are the Chinese practices of Tai Chi and Qi Gong. It may be noted that this is not an exhaustive list, and many other meditation techniques may also be practised by people worldwide. However, most meditations may have some or other processes given above, or even a combination of them (Hussain \& Bhushan, 2010).

Different meditation techniques may have different benefits, suit different people or suit the same person in a different growth stage. Often, a combination of techniques is also present in diverse spiritual traditions. Many people practice a combination of these techniques. These techniques do not exist in isolation, especially in the context of their origin. They are related to various assumptions about the nature of reality, the place of an individual in the larger scheme of things, and an ethical way of living. 


\section{Self-Assessment Questions}

Q31. Various techniques to control the mind are known as meditation - True/ False

Q32. This famous Swami .... had demonstrated voluntary control over multiple bodily organs in a lab in USA.

Q33. Concentrative Meditation is also known Samatha meditation - True/ False

Q34. Mindfulness meditation is also understood as process of cultivating Sakshi bhava - True/ False

Q35. In modern times, Maharshi .... started the Transcendental meditation movement.

Q36. Focusing on a single point is known as .....

Q37. Meditation where we remain aware of our thoughts, feelings and sensations in the present moment is known as ....

Q38. The interpersonally oriented meditations attempt to cultivate, maitri, mudita, .... And upeksha.

Q39. Yogasana, Tai Chi and Qi Gong are also considered body-based meditation - True/ False

\subsection{Meditative States in Patanjali Yogasutra}

Meditation, as described in Patanjali Yogasutras is mainly of the concentrative type, with narrowing and stabilizing of attention during the Dharana-Dhyana stages. After developing an unbroken flow of attention, one may be ready for the next stage of Samadhi, where experiences an absorption in the object of attention. He has further described various kinds and levels of Samadhi:

4.3.1. Samprajnata samadhi: These are lower levels of samadhi, obtained through thoughts, such as absorption on gross thought, subtle thoughts, bliss, or the essential individuality or Iness. 
4.3.2. Asamprajnata Samadhi: This is higher level of samadhi, which occurs when one can go beyond thoughts.

He has further differentiated between five types of Samadhi based on the quality of mind during those stages:

4.3.3. Savitarka Samadhi: in this stage, there is still a mixing of words, meaning and knowledge of an object.

4.3.4. Nirvitarka Samadhi: here, only the object is illuminated, without any gross thoughts.

4.3.5. Savichara Samadhi: in this samadhi, there is the presence of subtle thoughts.

4.3.6. Nirvichara Samadhi: in this state there is an absence of even subtle thoughts.

4.3.7. Nirbija Samadhi: this samadhi occurs when even the seeds of thoughts are restrained.

As one achieves the higher stages, one develops discriminative wisdom and the highest samadhi.

4.3.8. Dharma-Megha Samadhi: with further spiritual progress, Dharma-Megha Samadhi dawns, leading to oneness with the highest universal consciousness or Kaivalya, the soul's liberation.

\subsection{Effects of Meditation}

4.4.1. Benefits of Meditation: There has been an explosion of research on meditation since 1970, using both psychological and physiological measures. These studies have been done in advanced meditations with many years of practice and novice, beginner meditators. Similarly, it has been explored both in healthy populations and those suffering mental or physical illnesses.

In a review of the research (Hussain \& Bhushan, 2010), meditation leads to multiple effects on the autono-mic nervous system, slowing heart rate, decreased oxygen use, and lowered blood 
pressure. Other common findings related to meditation are increased alpha waves in the brain correlated with deep relaxation and theta waves associated with peace and blissful feeling, along with reduced activity in the brain's emotional centres. Improved attention, perceptual abilities, learning and memory, emotional maturity, and resilience wisdom also seem to be present.

Other reviews (Goyal, et al., 2014) focusing on meditation studies in the clinical population have reported a decrease in stress and anxiety, rumination, trauma symptoms, depression, deaddiction, and improvement in sleep, quality of life, well-being, self-compassion, and psychological flexibility. Studies on meditation's effect on physical health have commonly found improvement in hypertension, cardiovascular disorders, respiratory disorders, musculoskeletal diseases, better management of pain, headache, skin issues and improved immune response (Hussain \& Bhushan, 2010).

4.4.2. Need for caution: It is also essential to take a cautious approach (Fjorback \& Walach, 2012). Some studies have indicated specific adverse effects, often related to distress due to the resurfacing of old traumatic memories or unpleasant thoughts. Further, with those diagnosed with psychotic disorders, one needs a clinician's judgement before introducing meditation. A thoughtful choice needs to be made. There may also be individual responses to meditation, and personal guidance from a trained mentor is desirable.

\subsection{Modern Psychological Interventions Integrating Meditation}

Due to various benefits, meditation has increasingly been integrated with counselling and psychotherapy over the last few decades. It began with Benson's relaxation response and became popular with Jon Kabat-Zinn's Mindfulness-Based Stress Reduction Program. Subsequently, an entire trend of integrating meditation with modern psychology started, with examples like Mindfulness-Based Cognitive Therapy, Mindfulness-Based Relapse Prevention 
etc. (Fjorback \& Walach, 2012). The classic Mindfulness-Based Stress Reduction Program is conducted in a group format across eight weeks. One needs to engage in daily individual meditation and other homework for at least 30 minutes and a weekly group meeting of 2.5 hours.

In India too there has been a lot of research and application of yoga and meditation in the health setting (Kumar, Bhide, Arasappa, Varambally, \& Gangadhar, 2021). Given the variety of meditation-contemplation and philosophical systems available in the country with both many experts in these systems as well as a population open to these systems, one may find rich literature in this area. Sattva Enhancement Therapy and Sattva Cultivation Program is one such module developed at National Institute of Mental Health and Neuro Sciences, Bengaluru which integrated yoga psychology and meditation for improving mental health (Agrawal, 2021).

It is also important to remember that at the core of these traditions lies the belief that one can live a life with inner freedom, and it needs to be experienced first-hand and personally. Therefore, a global movement is happening now where psychologists themselves are engaging in yoga and meditation to experience its benefits before teaching it to others.

\section{Self-Assessment Questions}

Q40. Samprajnata samadhi are obtained by going beyond through thoughts - True/ False

Q41. An absence of even subtle thoughts are known as .... Samadhi.

Q42. Meditation leads to higher emotional maturity - True/ False

Q43. Meditation is not helpful in cardiovascular disorders and respiratory disorders - True/ False

Q44. One needs to use caution in applying meditation in ... disorders 
Q45. Jon Kabat-Zinn had developed Mindfulness-Based .... Program.

\subsection{Let us Sum Up}

In current times, there is a need to focus on positive mental health, and explore various indigenous models for comprehensive mental health care. In this context there is a need to study yoga and meditation traditions, which has been an important Indian contribution to the world. Yoga has been defined as a combination of spiritual techniques for finding existential freedom, or as the specific school of philosophy described in Patanjali Yogasutra. As per the former definition, one can study the three major Yogic paths of Bhakti yoga, Jnana yoga and Karma yoga. While one may find a description of causes of suffering, ways to release from suffering, and description of Kriya-yoga and Patanjali's Ashtanga yoga of Patanjali.

Meditation is a group of techniques for training the mind. Although it goes back in history, the modern research has expanded over the last few decades. the broad categories of meditation are focused attention or concentrative meditation, mindfulness or open awareness meditation, transcendental meditation and interpersonally oriented meditations. Other common forms of meditations are Japa, Mantra meditation, Yoga Nidra, Shavasana etc. Patanjali has also described various meditative states in his Yogasutra. Various benefits of Meditation have been found along with a few cautionary notes in recent research. Currently meditation is increasingly integrated with counseling and psychotherapy, and this is the way forward for developing positive mental health amongst in modern times.

6.0. Key Words: Yoga, Meditation, Positive Mental Health, India

\subsection{Answers to Self-Assessment Questions}

Q1. Depression 
Q2. False

Q3. True

Q4. Third

Q5. Liberation

Q6. Bhakti, Jnana, Karma

Q7. False

Q8. True

Q9. Bhagavad Gita

Q10. Four

Q11. True

Q12. False

Q13. True

Q14. svadhyaya, Tapa

Q15. antaranga

Q16. False

Q17. detachment (vairagya)

Q18. despair, irregular breathing.

Q19. Pratipaksha Bhavana.

Q20. Creation, maintenance, dissolution 
Q21. Asmita, Raga, Dwesha

Q22. True

Q23. attenuated, manifest

Q24. Dhyana

Q25. Asteya, Aparigraha

Q26. Santosha, Tapa

Q27. False

Q28. False

Q29. Pratyahara

Q30. Samyama

Q31. True

Q32. Swami Rama

Q33. True

Q34. True

Q35. Maharshi Mahesh Yogi

Q36. Focused Attention or Concentrative meditation

Q37. Mindfulness or Open awareness (open monitoring)

Q38. Karuna

Q39. True 
Q40. False

Q41. Nirvichara Samadhi

Q42. True

Q43. False

Q44. Psychotic

Q45. Stress Reduction

\subsection{Unit End Questions}

1. Discuss the limitations of routine mental health care and need for mental health promotion.

2. What is the role of yoga and meditation for positive mental health?

3. Write an essay on Patanjali's Ashtanga Yoga.

4. Write a short note on Klesha model of suffering as given in Patanjali yogasutra.

5. Describe types of meditation, and explain what are the benefits of regular meditation.

\section{Reflections}

1. What is your personal framework about life? (Answers to questions such as, who am I/ why am I born / what is my place in the universe / what is the best way to live?). Write it down. Remember these frameworks can keep changing and evolving.

2. In whatever you do, what are the topmost sources of motivation for you? Are there things which you do without focusing on the outcomes, without having too strong expectations? What are those? 
3. Would you consider something beyond yourself, which might be considered as transcendental, is personally relevant (A higher power, Divine/ Universe/Nature/Deepest Self). If yes, how would you describe this relationship?

\section{Let us apply}

4. Sit quietly for the next 5 minutes and try to notice whatever comes up in your mind. Later check how often these thoughts were related to either of the chitta-vrittis: (a) Reality-based thinking, (b) Misconceptions, (c) Imaginations of future, (d) Memory of past, or (e) Sleepy or dull states.

5. When you decide to change something within you and grow personally (for example, you will control your anger or use of social media), how often do you remember this decision and stick to it? How much faith do you have in yourself that you will do what you originally planned? How do you handle difficulties on the way, leave it quickly or face it with courage?

6. Since Pranava (AUM or Om sound) is considered to be the sound of Supreme Consciousness (Iswara), try chanting A, U, M for 5-10 minutes and write down your experience of this Japa meditation.

7. Developing the qualities of unconditional friendliness and loving-kindness and (Maitri), compassion (Karuna), sympathetic and appreciative joy (Mudita), and non-reactivity and equanimity (Upeksha), are meant to overcome suffering, despair, bodily instability, and irregular breathing. Try meditating upon 'Sarve bhawantu sukhina'/ May everyone be happy 
and visualise people around you, including yourself being happy. Then extend this happiness to every living being without any limits to it.

You can also do this exercise with others in a joint and creative manner, where each participant shares a good wish which they would like to send (for example, may there be good health in the world, may the plants in the garden bloom ...etc.).

\subsection{References}

Agrawal, J. (2021). Sattva Enhancement Therapy: An illustrative report. Indian Journal of Clinical Psychology, 48(2), 3-6.

Agrawal, J., \& Cornelissen, M. (2021). Yoga and Positive Mental Health. In S. G. Shivarama Varambally, Art and Science of Yoga in Mental and Neurological Healthcare A Manual.

Agrawal, J., \& Sahota, P. B. (2021). Interpersonal Aspects of Mindfulness and Concentrative Meditations. In S. K. Gupta, Handbook of Research on Clinical Applications of Meditation and Mindfulness-Based Interventions in Mental Health (pp. 237-254). IGI Global, USA.

Bockting, C. L. (2015). A lifetime approach to major depressive disorder: the contributions of psychological interventions in preventing relapse and recurrence. Clinical psychology review,. 41, 16-26.

Cardoso, R., deSouza, E., Camano, L., \& Leite, J. R. (2004). Meditation in health: an operational definition. Brain Research Protocols, 58-60. 
Christopher, J. C. (2008). Positive psychology, ethnocentrism, and the disguised ideology of individualism. Theory \& psychology,, 18(5), 563-589.

Fjorback, L. O., \& Walach, H. (2012). Meditation based therapies-a systematic review and some critical observations. Religions,, 3(1), 1-18.

Goleman, D., \& Davidson, R. (2017). The science of meditation: How to change your brain, mind and.

Goyal, M., Singh, S., Sibinga, E. M., Gould, N. F., Rowland-Seymour, A., Sharma, R., \& ... \& Haythornthwaite, J. A. (2014). Meditation programs for psychological stress and wellbeing: a systematic review and meta-analysis. JAMA internal medicine,, 174(3), 3.

Hofmann, S. G. (2010). The empirical status of the "new wave" of cognitive behavioral therapy. Psychiatric Clinics,, 33(3), 701-710.

Huhn, M. T.-W. (2014). Efficacy of pharmacotherapy and psychotherapy for adult psychiatric disorders: a systematic overview of meta-analyses. JAMA psychiatry,, 71(6), 706-7.

Hussain, D., \& Bhushan, B. (2010). Psychology of meditation and health: Present status and future directions. International Journal of Psychology and Psychological Therapy,, $10(3), 439-451$.

Kumar, V., Bhide, S. R., Arasappa, R., Varambally, S., \& Gangadhar, B. N. (2021). 'Soother of mind'-meditation in psychiatric disorders: a narrative review. BJPsych Advances, $1-7$.

Kutz, H. L. (1985). Meditation as an adjunct to psychotherapy. Psychotherapy and psychosomatics,, 43(4), 209-218. 
Larson, G. J. (2016). The Encyclopedia of Indian Philosophies Volume XII, Yoga: India's Philosophy of Meditation. Motilal Banarsidass.

Layous, K. C. (2011). Delivering happiness: Translating positive psychology intervention research for treating major and minor depressive disorders. The Journal of Alternative and Complementary.

Lippelt, D. P., Hommel, B., \& Colzato, L. S. (2014). Focused attention, open monitoring and loving kindness meditation: effects on attention, conflict monitoring, and creativity-A review. Frontiers in psychology,, 1083(5).

Lomas, T. \&. (2016). Second wave positive psychology: Exploring the positive-negative dialectics of wellbeing. Journal of Happiness Studies, , 17(4), 1753-1768.

Lomas, T. W. (2020). Third wave positive psychology: broadening towards complexity. . The Journal of Positive Psychology, , 1-15.

Lynch, J., Prihodova, L., Dunne, P. J., Carroll, A., Walsh, C., McMahon, G., \& White, B. (2018). Mantra meditation for mental health in the general population: A systematic review. European Journal of Integrative Medicine,, 23, , 101-108.

Rao, K. R., \& Paranjpe, A. C. (2016). Psychology in the Indian tradition. Springer. doi:10.1007/978-81-322-2440-2.

Reddy, M. (2010). Depression: the disorder and the burden. Indian journal of psychological medicine, 32(1), 1-2. Retrieved from https://doi.org/10.4103/0253-7176.70510

Sin, N. L. (2009). Enhancing well-being and alleviating depressive symptoms with positive psychology interventions: A practice-friendly meta-analysis. Journal of clinical psychology,, 65(5), 467-487. 
Suh, E. M. (2008). Comparing subjective well-being across cultures and nations. . The science of subjective well-being, , 414-423.

SURVEY, N. M. (2015-16). Retrieved from http://www.indianmhs.nimhans.ac.in/

Taimini, I. K. (1961). Science of Yoga. Quest Books.

Thomas, J. W., \& Cohen, M. (2014). A methodological review of meditation research. Frontiers in psychiatry,, 74(5).

Vázquez, C. H. (2009). Psychological well-being and health. Contributions of positive psychology. Annuary of clinical and health psychology,, 5, 15-27.

Walsh, R., \& Shapiro, S. L. (2006). The meeting of meditative disciplines and Western psychology: A mutually enriching dialogue. The American Psychologist,, 61(3), 227239. . doi:doi:10.1037/0003-066X.61.3.227

WHO. (2001). Mental Health : New Understanding, New Hope.

WHO. (2010). Global Health Estimates: Life expectancy and leading causes of death and disability. Retrieved from https://www.who.int/data/gho/data/themes/mortality-andglobal-health-estimates 